\title{
Electrohydrodynamic behavior of water droplets on a Horizontal Super Hydrophobic Surface and its Self-cleaning Application
}

Jian $\mathrm{Li}^{*}$, Yuan Wei, Zhengyong Huang, Feipeng Wang, Xinzhu Yan, Zhuolin Wu

State Key Laboratory of Power Transmission Equipment \& System Security and New Technology, Chongqing University, Chongqing 400044, China

*Corresponding author. lijian@cqu.edu.cn 


\section{ABSTRACT:}

Moisture is a significant factor that affects the insulation performance of outdoor high-voltage insulators in power systems. Accumulation of water droplets on insulators causes severe problems such as flashover of insulators and power outage. In this study, we develop a method to fabricate a micro/nano hierarchical super hydrophobic surface. The as-prepared super hydrophobic surface exhibits a water contact angle (WCA) of $160.4 \pm 2^{\circ}$, slide angle (SA) less than $1^{\circ}$ and surface free energy (SFE) of $5.99 \mathrm{~mJ} / \mathrm{m}^{2}$. We investigated the electrohydropdynamic behavior of water droplet on a horizontal super hydrophobic surface compared with hydrophobic RTV silicone rubber surface which was widely used as anti-pollution coating or shed material of composite insulator. Results show that water droplet tended to a self-propelled motion on the super hydrophobic surface while it tended to elongate and break up on the RTV surface. The micro/nano hierarchical surface structure and chemical components with low surface free energy of the super hydrophobic surface jointly contributed to the reduction of skin fraction drag and subsequently made it possible for the motion of water droplet driven by electric field. Furthermore, the self-propelled motion of water droplets could also sweep away contaminations along its moving trace, which provides super hydrophobic surface a promising anti-pollution prospect in power systems.

Keywords: Micro/nano hierarchical structure, Superhydrophobicity, RTV silicone rubber, Electrohydrodynamic behavior of water droplets, Self-cleaning 


\section{Introduction}

Moisture is a significant factor that affects the insulation performance of outdoor high-voltage dielectrics in power systems [1, 2]. Fog, dew and drizzle deposit on dielectric surface reduce the surface dry area and cause a significant decrease in electric insulation strength, which may lead to severe problems such as flashover of power insulator strings, damage of power transformers and even an electric power outage [3-5].

Room temperature vulcanized (RTV) silicone rubber with water repellency property (water contact angle greater than $90^{\circ}$ ) could prevent the surface from being completely wetted and owns anti-pollution ability resulting from migration of low molecular weight (LMW) silicon components from the bulk to the surface [6,7], which has been widely used in China, Canada and the United States for the past three decades [8-10]. However, considerable applications and studies [11-14] have indicated that hydrophobicity of RTV silicone rubber decreased with the increase of serving time. Moreover, the water droplet slide angle (SA) of RTV silicone rubber is pretty large (greater than $10^{\circ}$ ). Discrete water droplets are largely sessile and not easy to slide off which may result in partial discharge along the coating surface and accelerate degradation and aging of the RTV silicone rubber. In addition, RTV silicone rubber exhibits a large adhesion force to impurity particles due to electrostatic interaction and surface viscosity $[15,16]$, which reduces its anti-pollution performance.

Inspired by lotus leaves in nature, super hydrophobic surfaces with high water contact angle (WCA greater than $150^{\circ}$ ) and low contact angle hysteresis (CAH less than $5^{\circ}$ ) have recently drawn considerable attentions in research and applications towards anti-icing [17, 18], anti-frosting [19, 
20], drag reduction [21, 22], self-cleaning [23, 24] and so forth. Many methods such as chemical etching [25, 26], electrochemical deposition [27, 28], electrospinning [29, 30], phase separation $[31,32]$, plasma treating $[33,34]$ and sol-gel process $[35,36]$ have been adopted to mimic the "lotus-effect" super hydrophobic surfaces. The mainly principle of these methods is a combination of low surface energy composition and micro/nano hierarchical surface structure [37, 38]. The super hydrophobic surfaces allow a large amount of air entrapment and thus consequently reduce skin friction drag between the surface itself and the droplet $[39,40]$. Water droplets tend to roll or slide off the surfaces by tilting or external vibration. The excellent hydrophobicity and self-cleaning property of super hydrophobic surface are beneficial to reduce water droplets and contaminations accumulation, which therefore make super hydrophobic material a prospective candidate to improve anti-pollution performance of outdoor high-voltage insulators in power systems.

In this work, we designed a simple process to fabricate super hydrophobic surface based on nano-sized silica $\left(\mathrm{SiO}_{2}\right)$ particles and perfluoroalkyl methacrylic copolymer (PMC). The as-prepared super hydrophobic exhibits coral-like hierarchical surface structure with a WCA of $160.4 \pm 2^{\circ}$ and SA less than $1^{\circ}$. The electrohydrodynamic behaviors of water droplets located on the super hydrophobic surface and RTV silicone rubber surface were investigated by applying an ac electric field. A self-propelled motion of water droplets on super hydrophobic surface was observed while an elongation and breakup behavior of water droplets on RTV silicone rubber surface was found. The differences in electrohydrodynamic behaviors of two surfaces were analyzed from the differences in surface hydrophobicity. Furthermore, the self-propelled motion of water droplet on super hydrophobic surface could sweep away both water-soluble and 
non-water-soluble contaminations along its moving trace, which provides feasibility for anti-pollution application of super hydrophobic surface served in power systems.

\section{Experiments}

\subsection{Materials}

Perfluoroalkyl methacrylic copolymer (PMC, Dupont Co., Ltd., USA) and hydrophobic nano silica particles (7-40 nm in diameter, Aladdin Co., Ltd., China) were obtained commercially and used as received. The acetic acid and ethyl alcohol (Tianjin Baishi Chemical Co., Ltd., China) were chosen as solvents. A kind of commercial product room temperature vulcanized silicone rubber coating (RTV, Tengzhou Zhixing Co., Ltd., China) was obtained as a control group. Pure $(99.5 \%)$ quartz glass plates $(10 \mathrm{~cm} \times 10 \mathrm{~cm} \times 0.3 \mathrm{~cm})$ were used as substrates. Permanganate particles (KMnO4, Guanrui Biomart Co., Ltd., China) were used as the contaminants.

\subsection{Preparation of super hydrophobic surface}

Processes to prepare the super hydrophobic surface were mainly consisted of two steps. The first step was the preparation of a precursor nanocomposite suspension. Perfluoroalkyl methacrylic copolymer $(5 \mathrm{~mL})$ was diluted with acetic acid $(50 \mathrm{~mL})$ and the solution was stirred at $200 \mathrm{r} / \mathrm{min}$ and room temperature for 10min. Meanwhile, hydrophobic silica nanoparticles (3g) were dispersed in ethyl alcohol $(20 \mathrm{~mL})$ and ultrasonic sonication for $10 \mathrm{~min}$. Then the PMC solution and silica nanoparticles dispersion were mixed and ultrasonically dispersed for $1 \mathrm{~h}$ to obtain a homodisperse nanocomposite suspension. The second step was the formation of super hydrophobic surface. Glass substrates were cleaned with deionized water and drying, after which the silica-PMC suspension was spray deposited onto the glass substrates by an airbrush with a nozzle diameter and compressed air pressure of $0.5 \mathrm{~mm}$ and $150 \mathrm{KPa}$, respectively. Then the samples were kept at 
$120^{\circ} \mathrm{C}$ for $2 \mathrm{~h}$ to remove the residual solvents. Finally, the super hydrophobic surface was fabricated. The thicknesses of the super hydrophobic coatings were measured at $0.05 \pm 0.01 \mathrm{~mm}$ by a coating thickness gauge (Qnix 4500, Automation Dr. Nix GmbH \& Co.KG, Germany).

The RTV silicone rubber coating with red color used in the experiments was industrial grade product and it was applied on the glass substrate using dip-coating method. The samples dried in room temperature for $24 \mathrm{~h}$ before hydrophobicity test. The thicknesses of the RTV silicone rubber coatings were measured at $0.12 \pm 0.01 \mathrm{~mm}$.

\subsection{Method}

\subsubsection{Characterizations}

The surface morphology of the samples was observed by a field emission scanning electron microscope (FE-SEM, SU-8010, Hitachi Co., Ltd., Japan) equipped with an energy dispersive X-ray spectroscopy (EDS). Prior to FE-SEM characterization, the samples were coated with a thin layer of gold to reduce surface charging. EDS measurements were employed to characterize the surface chemistry of the as-prepared surfaces. The surface topography of the sample was observed by an atomic force microscope (AFM, XE7, Park Systems Co., Ltd. South Korea) at ambient conditions and room mean square roughness $\left(\mathrm{R}_{\mathrm{rms}}\right)$ of the samples was evaluated via the AFM apparatus software XEL.

Water contact angles (WCAs) of the samples were measured with an optical contact angle meter (Drop Meter A-200, MAIST Vision Inspection \& Measurement Co., Ltd., China) by sessile drop method. In this method the WCA is formed between the liquid/solid interface and liquid/air interface. A micrometric syringe was used to dispense a $10 \mu \mathrm{L}$ deionized water droplet on the sample surfaces and five measurements were performed on different areas of each sample to 
eliminate test error. The slide angle (SA) was defined as the slope angle when the water droplet began to roll off the gradually tilting surface and the sequence photographs of the sliding behavior of water droplet on inclined surface were taken every $16 \mathrm{~ms}$ by the contact angle meter equipped with a computer-controlled camera.

\subsubsection{Electrohydrodynamic behavior test}

Electrohydrodynamic behaviors of water droplets on the super hydrophobic surface and RTV silicone rubber surface were tested by applying a tangential oscillating electric field (frequency $50 \mathrm{~Hz}$ ) along the surfaces. The sample was cleaned with ethyl alcohol to eliminate any dust particles before placed exactly horizontally to exclude surface inclination. A drop of water was dropped on the center of the sample surface, the volume of which was controlled by a micropipette. A camera (Canon EOS 70D, with an EF-S macro lens) was employed to record the water droplet behavior as the applied electric field increased.

\subsubsection{Self-cleaning test}

A self-cleaning test was established to evaluate the feasibility of self-cleaning effect of the prepared super hydrophobic surface by applying a tangential electric field. Permanganate $\left(\mathrm{KMnO}_{4}\right)$ particles, clay particles and sand particles were selected to simulate water-soluble and non-water-soluble contaminants respectively. The test procedure and method were the same as electrohydrodynamic behavior test. Self-cleaning effect resulting from water droplets movement driven by electric field was evaluated through the amount of surface contamination before and after droplet falling off the super hydrophobic surface. 


\section{Results and discussion}

\subsection{Surface characterization}

Fig. 1a and 1b showed the FE-SEM images of the as-prepared super hydrophobic surface and RTV silicone rubber surface, respectively. In Fig. 1a, numerous coral-like microstructures were observed on the as-prepared super hydrophobic surface. It was found that these coral-like microstructures with length about several micrometers were randomly distributed at low magnification $(\times 5.0 \mathrm{~K})$. Nanoparticles with diameters in a range of tens of nanometers were observed on the coral-like microstructure at high magnification $(\times 50.0 \mathrm{~K})$. A micro and nano hierarchical structure of the as-prepared super hydrophobic surface was observed. There also appeared several caves between coral-like microstructures which provided the possibility to allow air to fill in. The formation of air layer was beneficial to the excellent hydrophobicity of super hydrophobic surface. While in Fig. 1b, a relatively flat surface topography was observed on RTV silicone rubber surface at both low and high magnification.
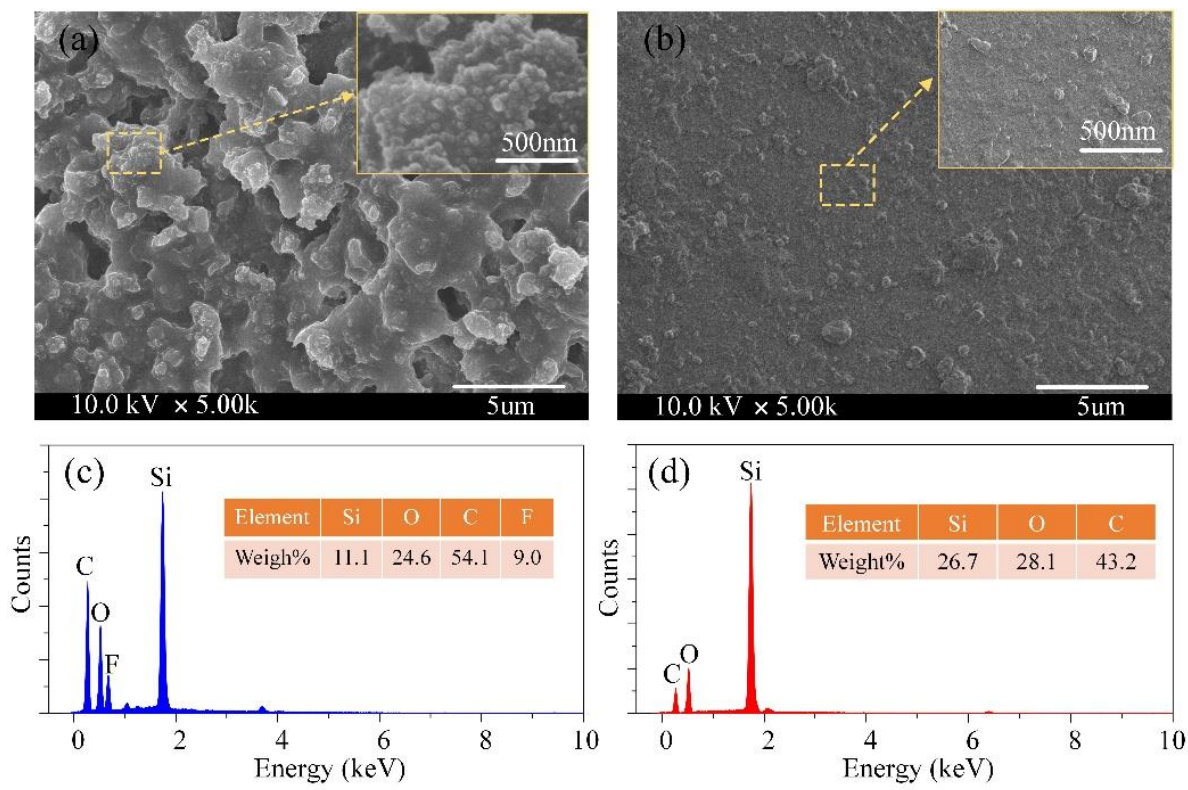

Fig. 1. FE-SEM images and EDS spectra of the super hydrophobic surface (a,c) and RTV silicone rubber surface $(b, d)$. 
Energy dispersive X-ray spectroscopy (EDS) was used to analyze chemical composition of the two different surfaces. The EDS spectra of the as-prepared super hydrophobic surface in Fig. 1c revealed the coating mainly consists of $\mathrm{C}, \mathrm{O}, \mathrm{Si}$ and $\mathrm{F}$ elements with a weight ratio at 54.1:24.6:11.1:9.0. The appearance of $\mathrm{F}$ element indicated that nano silica particles were low surface energy modified by fluorine-containing polymer. The EDS spectra of the RTV silicone rubber surface in Fig. 1d revealed the coating consists of $\mathrm{C}, \mathrm{O}$ and $\mathrm{Si}$ elements with a weight ratio at 43.2:28.1:26.7. This is because the RTV silicone rubber is basically composed of polydimethylsiloxane (PDMS) macromolecular chains and closely packed methyl groups $\left(-\mathrm{CH}_{3}\right)$ on the branched chain of PDMS results in the hydrophobicity to some extent.
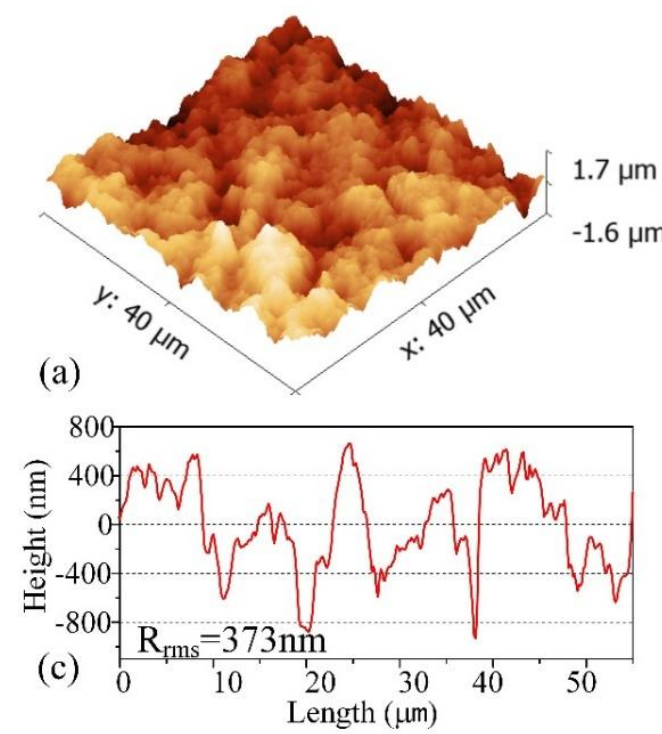

(b)
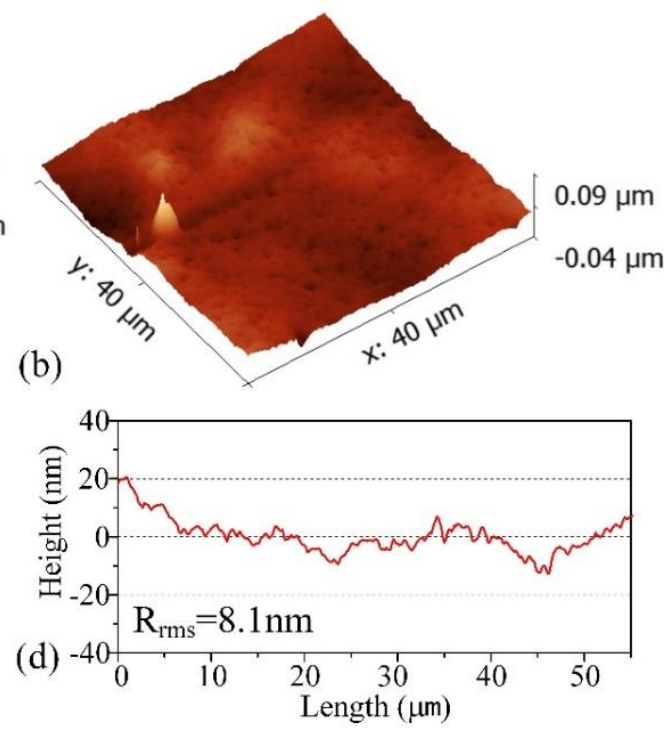

Fig. 2. AFM images and cross-section profile roughness curves of the super hydrophobic surface $(a, c)$ and RTV silicone rubber surface $(b, d)$.

The three-dimension surface morphologies (scan area $40 \times 40 \mu \mathrm{m}$ ) of the as-prepared super hydrophobic surface and RTV silicone rubber surface were shown in Fig. 2a and 2b, respectively. In Fig. 2a, the altitudes of surface microstructures varied between $-1.6 \mu \mathrm{m}$ to $1.7 \mu \mathrm{m}$ and exhibited hierarchical rough structures with random distribution of micro-size peaks and nano-size 
protuberances, which consisted with the results observed in Fig. 1a. The cross-section profile roughness curve was shown in Fig. 2c. The room mean square roughness $\left(\mathrm{R}_{\mathrm{rms}}\right)$ of the as-prepared super hydrophobic surface was calculated at $373 \mathrm{~nm}$. As a contrast, the three-dimension surface morphology of RTV silicone rubber was shown in Fig. 2b. The surface had a quite uniform morphologies with altitude varying from $-40 \mathrm{~nm}$ to $90 \mathrm{~nm}$, which verified the results in Fig. 1b. The $\mathrm{R}_{\mathrm{rms}}$ of cross-section of RTV silicone rubber was measured at $8.1 \mathrm{~nm}$, which was much smaller than that of the as-prepared super hydrophobic surface.

\subsection{Wettability}

The wettability of the as-prepared super-hydrophobic surface and RTV silicone rubber surface was measured based on edge detection by contact angle meter under indoor environment $\left(25^{\circ} \mathrm{C}\right.$, $\mathrm{RH}=40 \pm 10 \%$ ). The bare glass substrate exhibited a WCA of $37.5 \pm 2^{\circ}$ which was hydrophilic. The as-prepared super hydrophobic surface exhibited superhydrophobicity with a WCA of $160.4 \pm 2^{\circ}$ as shown in Fig. 3a. Water droplets with volume varying from $10 \mu \mathrm{L}$ to $60 \mu \mathrm{L}$ stood upon the super hydrophobic surface with spherical shape as shown in Fig. 3b. The RTV silicone rubber surface exhibited a WCA of $105.8 \pm 2^{\circ}$ as shown in Fig. 3c. The appearance of water droplets with volume varying from $10 \mu \mathrm{L}$ to $60 \mu \mathrm{L}$ were shown in Fig. $3 \mathrm{~d}$. 

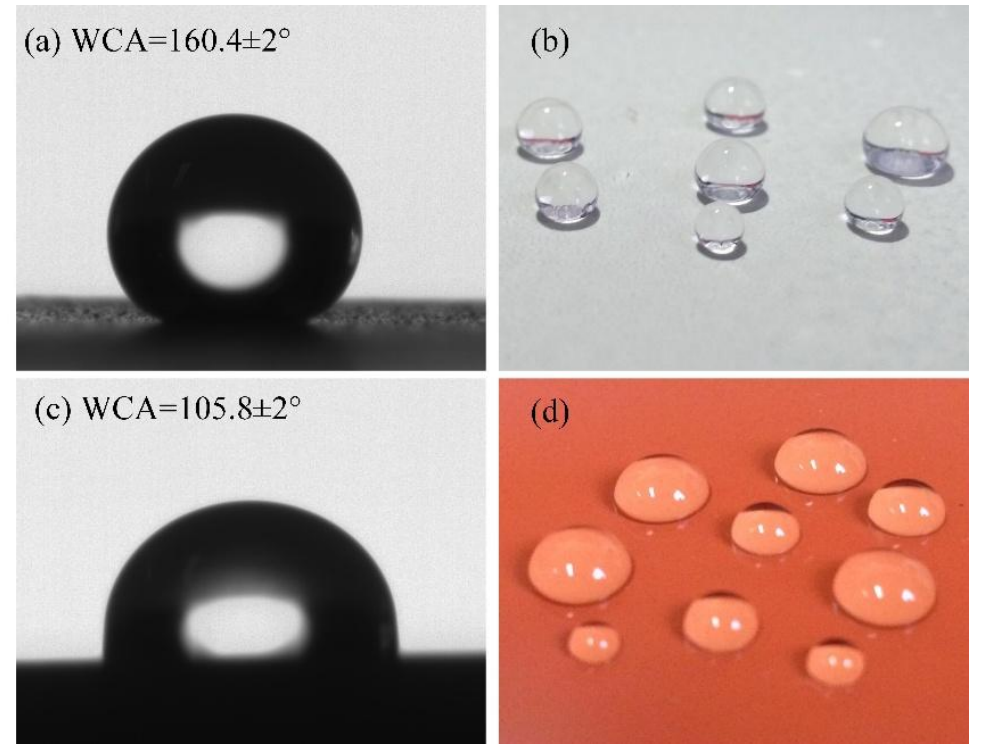

Fig. 3. Water droplet contact angles (WCAs) on the as-prepared super hydrophobic surface $(a, b)$ and RTV silicone rubber surface (c,d).

Secondly, the slide angle (SA) is another important criterion for super hydrophobic surface. As shown in Fig. 4a, a $10 \mu \mathrm{L}$ drop of distilled water was dropped onto the as-prepared super hydrophobic surface. The water droplet could easily slide off the surface when the inclination angle was less than $1^{\circ}$. The sequence photographs indicated that there was almost little drag resistance for the sliding of water droplets. As a contrast, the SA of RTV silicone rubber was also measured as shown in Fig. $4 \mathrm{~b}$. The $10 \mu \mathrm{L}$ droplet gradually began to move until the surface was tilted at about $21^{\circ}$. Moreover, the surface free energy (SFE) of the two surfaces was estimated by Owens-Wendt method [41, 42]. Deionized water and methylene diiodide were used as measuring liquids. The contact angles of $10 \mu \mathrm{L}$ methylene diiodide droplet on super hydrophobic surface and RTV silicone rubber surface were $118 \pm 2^{\circ}$ and $66 \pm 2^{\circ}$, respectively. Therefore the SFEs of the two surfaces were estimated at $5.99 \mathrm{~mJ} / \mathrm{m}^{2}$ and $25.48 \mathrm{~mJ} / \mathrm{m}^{2}$, respectively. Results indicated that the as-prepared super hydrophobic surface exhibited a greater water contact angle, much smaller slide angle as well as surface free energy. 


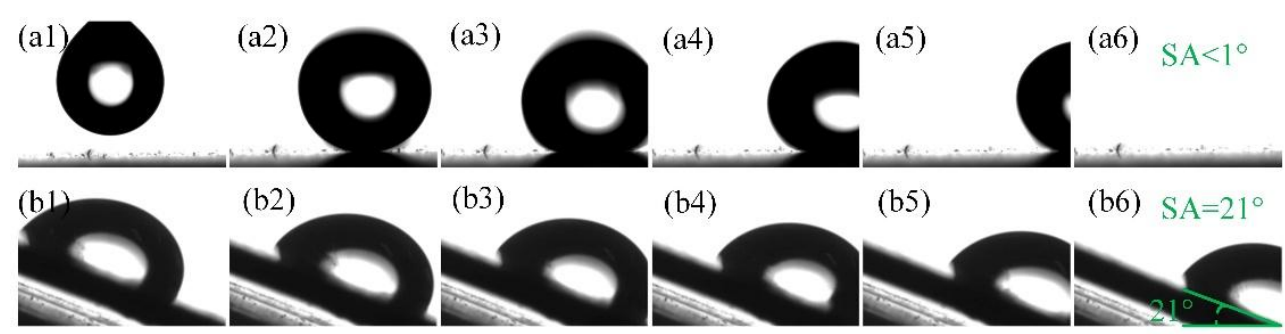

Fig. 4. Sequence photographs of $10 \mu \mathrm{L}$ water droplet sliding off the as-prepared super hydrophobic surface (a) and RTV silicone rubber surface (b).

To sum up, the superhydrophobicity of the as-prepared surface mainly results from the presence of hierarchical rough structures and composition with low surface energy. The as-prepared surface can entrap air cavities between micro- and nano- structures producing two different interfaces. One of them is formed between water and air where the friction resistance is significantly reduced. The other interface is formed between water and hierarchical structures, where the mainly friction resistance generates. A water droplet with a diameter much larger than the micro/nano structures is suspended on the rough structures rather than traps into the caves between the rough structures. The wettability of the heterogeneous rough surface was summarized by Cassie-Baxter's equation[43]:

$$
\cos \theta_{c}=f_{1} \cos \theta-f_{2}
$$

Where $\theta c$ and $\theta$ refer to the WCAs on the as-prepared super hydrophobic surface and smooth glass surfaces, respectively. The fractions of water/solid interface and water/air interface are presented by $f_{1}$ and $f_{2}$, respectively. Given that $f_{1}+f_{2}=1, \theta c=160.4^{\circ}$, and $\theta=37.5^{\circ}, f_{1}$ and $f_{2}$ are evaluated at 0.032 and 0.968 . The results indicates that approximately $96.8 \%$ of interface serves as the contact area of water/air interface, while the remaining $3.2 \%$ serves as the contact area of water/soild interface. Therefore, the as-prepared super hydrophobic surface owns excellent hydrophobicity as well as low skin drag resistance. 


\subsection{Electrohydrodynamic behavior of water droplet}

Fig. 5 showed the schematic diagram of electrohydrodynamic behavior test. Two cylindrical copper electrodes ( $1 \mathrm{~cm}$ in diameter and $9 \mathrm{~cm}$ in length) were embedded in the insulator separated by a distance of $8 \mathrm{~cm}$. One electrode was connected to an AC high voltage power source (maximum output voltage: $\mathrm{V}_{\mathrm{rms}}=50 \mathrm{kV}$ ) and the other was grounded. A $40 \mu \mathrm{L}$ drop of deionized water (conductivity, $20 \mu \mathrm{S} / \mathrm{cm}$ ) was dripped on the sample surface center using a micropipette. Then an oscillating voltage (frequency $50 \mathrm{~Hz}$ ) was applied between the cylindrical electrodes. An oscilloscope was set to monitor the voltage output, and the voltage increased uniformly at a rate of $1 \mathrm{kV} / \mathrm{s}$ until the water droplet began to mechanical instability.

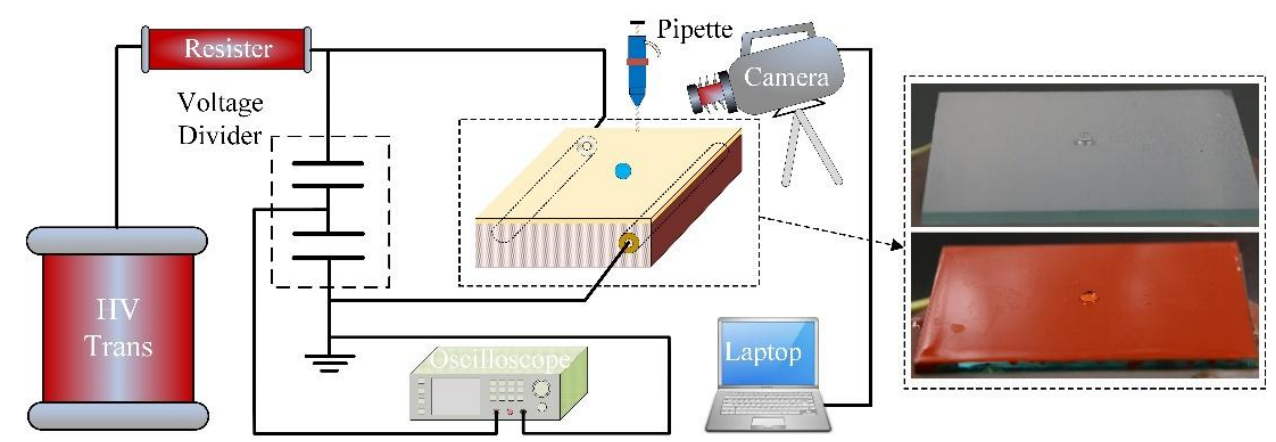

Fig. 5. The schematic diagram of electrohydrodynamic behavior test of water droplet.

Fig. 6 showed the eletrohydrodynamic behavior of water droplet on RTV silicone rubber surface when applying a tangential electric field (Supplementary Video.1). Initially, water droplet stood on RTV silicone rubber surface with a hemispheric shape as shown in Fig. 6a. When external electric field was applied, water droplet responded to the electric field and began to vibrate at its initial position. With the increase of electric field, water droplet gradually elongated along the direction 
of electric field and formed protuberance at the two tips as shown in Fig. 6b-6e. Once the electric field reached the threshold (about $3.2 \mathrm{kV} / \mathrm{cm}$, corresponding to electrode configuration and droplet volume), water droplet shifted to mechanical unstable and ejected water filaments from its tips as shown in Fig. 6f. Finally, water droplet split into several pieces by strong electric field as shown in Fig. 6i. The results revealed that RTV silicone rubber gradually lost its hydrophobicity when applying a strong tangential electric field.

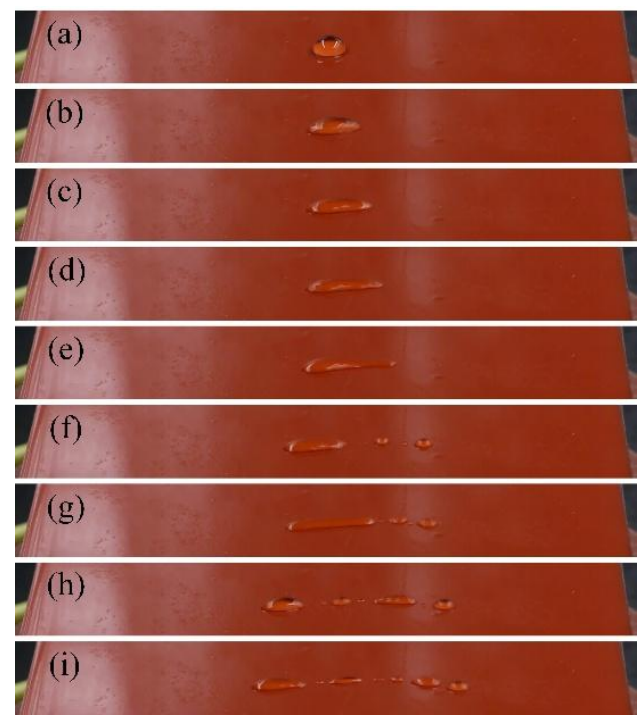

Fig. 6. Electrohydrodynamic behavior of water droplet on the RTV silicone rubber surface. (Supplementary Video.1)

Fig. 7 showed the electrohydrodynamic behavior of water droplet on super hydrophobic surface when applying a tangential electric field (Supplementary Video.2 and Video.3). In Fig. 7a, water droplet on super hydrophobic surface initially maintained its spherical shape due to the excellent hydrophobicity. When the external electric field was applied, the vibration and deformation phenomenon of water droplet responding to electric field was observed, but the water droplet still remained immobile on the coating surface. As the strength of the electric field increased, the water droplet destabilized and suddenly began to slide off from one side of the super hydrophobic surface once the electric field exceeded a threshold value (about $2.7 \mathrm{kV} / \mathrm{cm}$, corresponding to 
electrode configuration and droplet volume). The droplet remained intact during the moving process before it rolled off the surface as shown in Fig. 7a2-7a9. Water droplet sliding from another side of super hydrophobic surface was also observed and showed in Fig. 7b. Observation results showed that the movement path of the water droplet was always parallel to the direction of electric field in most cases.

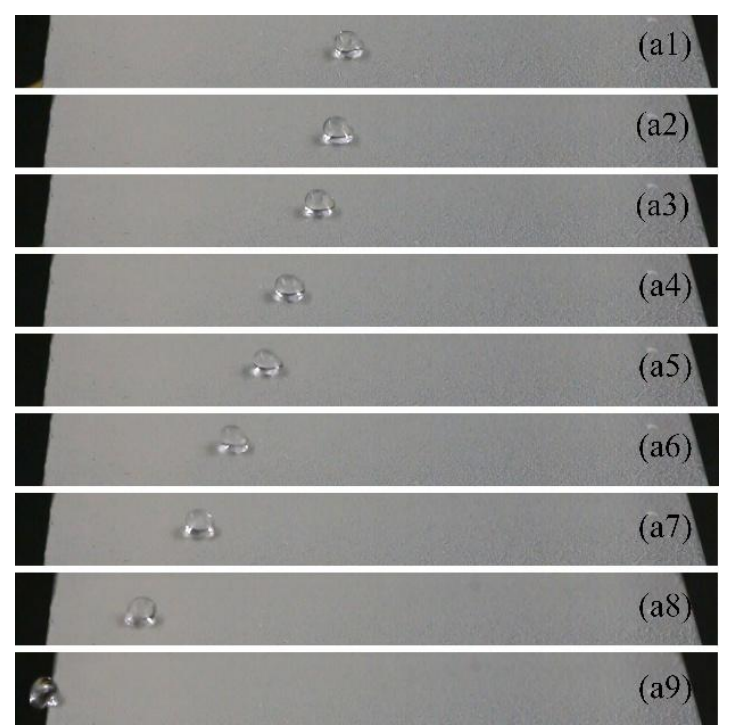

(a)

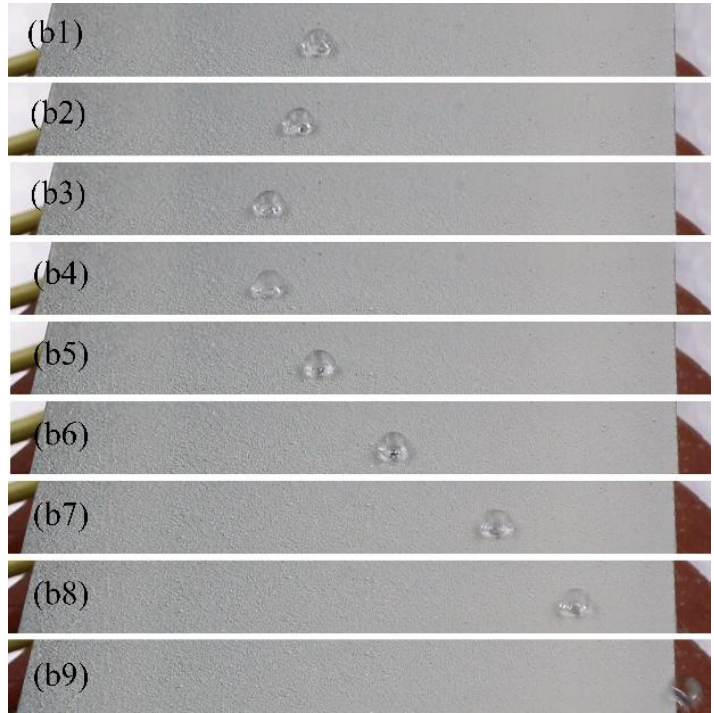

(b)

Fig. 7. Electrohydrodynamic behavior of water droplet on the super hydrophobic surface. (Supplementary Video.2 and Video.3)

The electrohydrodynamic behaviors of a water droplet on super hydrophobic surface and RTV silicone rubber surface were mainly due to the polarization and electrification phenomenon of water droplet. Fig. 8 showed the electric field distribution of a droplet on the super hydrophobic surface based on the experimental electrode setup. In Fig. 8a, a relative uniform electric field paralleling to the surface was generated by the two embedded electrodes. When a water droplet was placed on the center of the surface, the surrounding electric field changes, owing to the difference in material dielectric properties. The electric field was distorted and significantly enhanced at the droplet-surface-air contact area. The magnitude of the electric field at this area 
was almost ten times greater than that inside the droplet body as shown in Fig. 8c. The electric field was extremely non-uniform around the droplet and the high electric field strength potentially triggered a slight corona discharge at the droplet-air interface $[4,45,46]$, which mainly donated the net charge accumulation in the droplet body. Hence, a driven force from external electric field was exerted on the charged water droplet $[47,48]$ and the external electric field governed and driven the electrohydrodynamic behavior of a water droplet both on super hydrophobic surface and RTV silicone rubber surface.

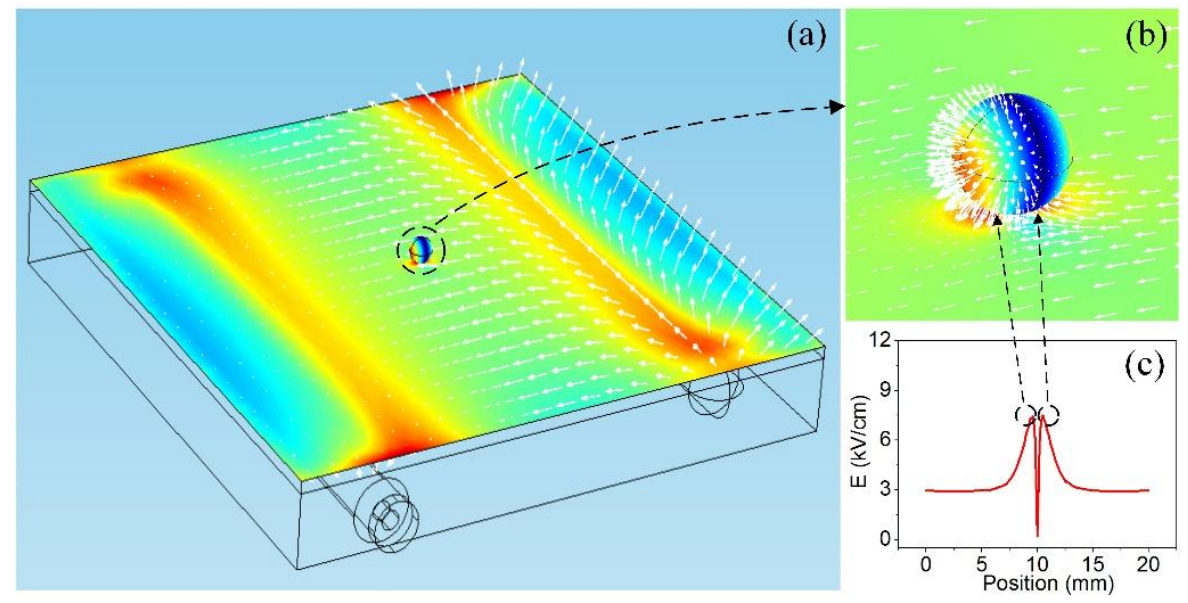

Fig. 8. Electric field simulation of water droplet on super hydrophobic surface.

The significant differences in electrohydrodynamic behaviors of water droplet on RTV silicone rubber and super hydrophobic surface are mainly due to the differences in surface hydrophobicity, which further result from the differences in surface free energy and microstructures of the two different surfaces. The super hydrophobic surface was made up of chemical components low surface free energy which resulted in a relative low attractive force between the surface and water droplet. Moreover, the micro and nano hierarchical structure of the super hydrophobic surface could lift up water droplet which replaced the droplet/coating interface with droplet/air interface 
and significantly reduced the skin drag resistance. The drag-reducing effect of super hydrophobic surface can be explained according to the Newton's Law of viscosity [44]:

$$
F=\mu A \frac{d u}{d y}
$$

Where $F$ refers to the drag force, $\mu$ refers to the dynamic viscosity of the water droplet, $A$ refers to the wetting area and $d u / d y$ refers to the velocity gradient in the water droplet. Considering $\mu$ is a constant for water droplet, the drag force $F$ is proportional to the wetting area $A$. Since the micro and nano hierarchical structure of the super hydrophobic surface produces an air layer between the droplet and solid surface, a decrease in wetting area is expected, which subsequently reduces the skin drag force between droplet and super hydrophobic surface. Hence, the water droplet on super hydrophobic surface exhibits a self-propelled motion driven by electric field, while the self-propelled motion behavior of water droplet on RTV silicone rubber is restricted.

\subsection{Electrohydrodynamic self-cleaning effect}

The self-propelled motion of a water droplet on a polluted super hydrophobic surface under a tangential electric field was also examined. In Fig. 9, a small quantity of water-soluble particles $\left(\mathrm{KMnO}_{4}\right.$ particles) were sprinkled uniformly on the surface to simulate soluble contamination. Initially, the droplet approached the particles as shown in Fig. 9a. Some particles dissolved in the droplet gave it a purple tinge. Subsequently, the droplet moved almost parallel to the electric field, as shown in Fig. 9b, and its color became deeper (Figs. 9c-9d). The droplet carried any particles along the moving trace away, leaving a clean track, as shown in Fig. 9e. Finally, the droplet fell off the surface edge, with dissolved contaminants in it. In Fig. 10, the electrohydrodynamic self-cleaning effect for non-water-soluble particles was also tested. Similarly, clay and sand 
particles with black and little yellow colors were sprinkled uniformly on the surface to simulate non-water-soluble contaminations. Driven by the electric field, water droplet on super hydrophobic surface gradually approached the contamination particles, attracted the particles and took them away along its moving trace as showed in Fig. 10a-10h. Combined with the electrohydrodynamic self-cleaning test results in Fig. 9, it was showed that water droplets driven by a tangential electric field could take away both water-soluble particles and non-water-soluble particles along the moving trace on super hydrophobic surface, which indicted the electrohydrodynamic self-cleaning feasibility for anti-pollution application in power system.

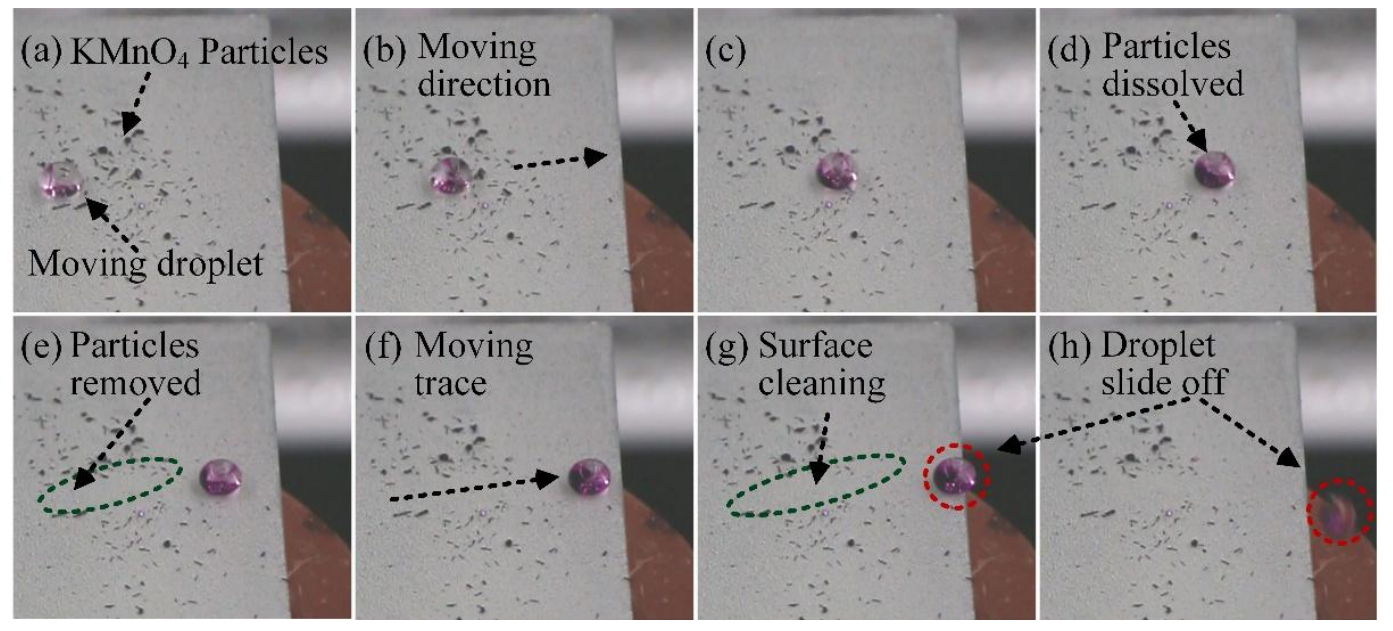

Fig. 9. Electrohydrodynamic self-cleaning effect for water-soluble particles $\left(\mathrm{KMnO}_{4}\right.$ particles $)$ on the horizontal super hydrophobic surface.

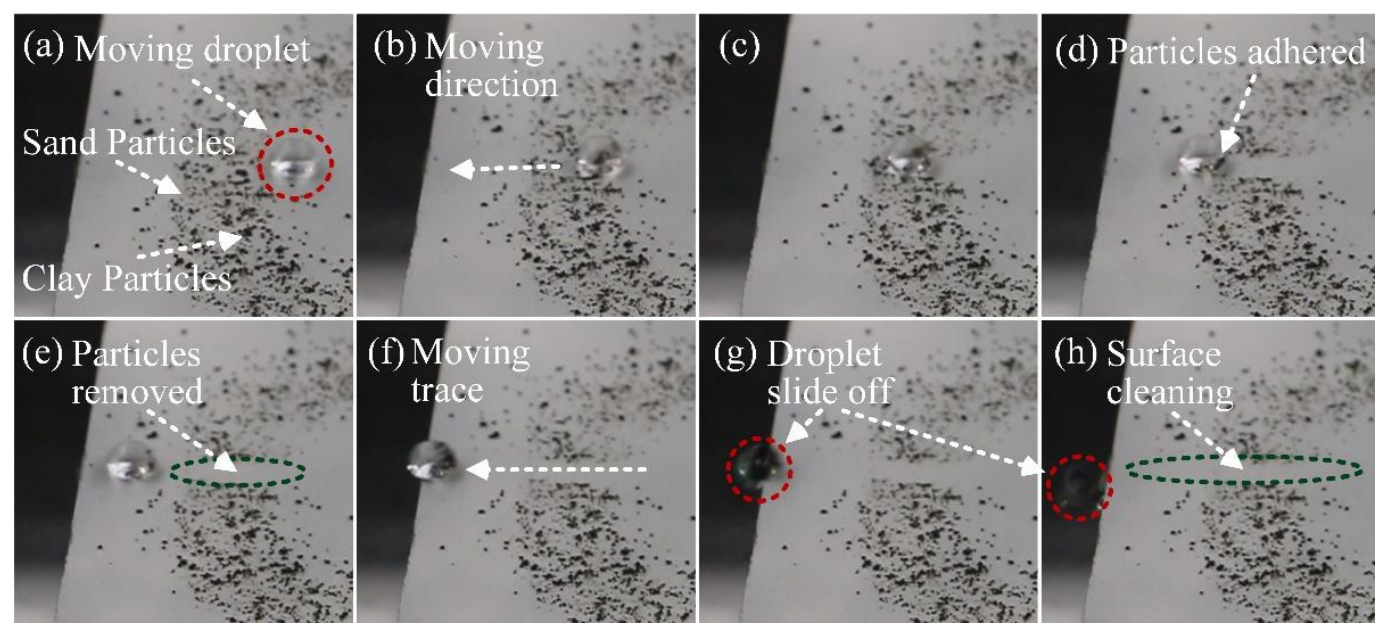


Fig. 10. Electrohydrodynamic self-cleaning effect for non-water-soluble particles (clay and sand particles) on the horizontal super hydrophobic surface.

\section{Conclusion}

A micro/nano hierarchical super hydrophobic surface was fabricated based on nano-sized silica $\left(\mathrm{SiO}_{2}\right)$ particles and perfluoroalkyl methacrylic copolymer (PMC). The as-prepared surface exhibited a water contact angle (WCA) of $160.4 \pm 2^{\circ}$, slide angle (SA) less than $1^{\circ}$ and surface free energy (SFE) $5.99 \mathrm{~mJ} / \mathrm{m}^{2}$. FE-SEM and AFM images shown that the as-prepared super hydrophobic surface exhibited a coral-like surface topography and much larger surface roughness compared with RTV silicone rubber surface. Water droplets exhibited different electrohydrodynamic behaviors on the super hydrophobic surface and hydrophobic RTV silicone rubber surface. When applying a tangential electric field, water droplet on super hydrophobic surface tended to a self-propelled motion while it tended to elongate and break up on RTV silicone rubber surface. The significant differences in electrohydrodynamic behaviors were due to the differences in surface microstructures and chemical components. The micro/nano hierarchical surface structure and low surface free energy components of super hydrophobic jointly contributed to the decrease of skin fraction drag and subsequently made it possible for the motion of water droplet driven by electric field. Moreover, the self-propelled motion of water droplet could sweep away both water-soluble and non-water-soluble contaminations along its moving trace. The present study demonstrated a promising way to achieve self-cleaning of super hydrophobic surface by applying a tangential electric field, which provided a potential anti-pollution application prospect in power systems. 


\section{Acknowledgment}

The authors acknowledge the National Science Foundation of China (No.51321063) and the

National Key Basic Research Program of China (973 program) (2015CB251003). The Project 111

of the Ministry of Education, China (No.B08036) is also appreciated for supporting this work.

\section{Reference}

[1] S.M. Gubanski, Modern outdoor insulation-Concerns and challenges, IEEE Trans. Dielectr. Electr. Insul. 21 (2005) 5-11.

[2] G.X. Xie, F. He, X. Liu, L.N. Si, D. Guo, Sessile multidroplets and salt droplets under high tangential electric fields, Sci. Rep. 6 (2016) 25002-25003.

[3] Y. Higashiyama, S. Yanase, T. Sugimoto, DC corona discharge from water droplets on a hydrophobic surface, J. Electrostat. 55 (2002) 351-360.

[4] Y. Zhu, K. Haji, M. Otsubo, C. Honda, N. Hayashi, Electrohydrodynamic behaviour of water droplet on an electrically stressed hydrophobic surface, J. Phys. D: Appl. Phys. 39 (2006) 1970-1975.

[5] S.M. Rowland, F.C. Lin, Stability of alternating current discharges between water drops on insulation surfaces, J. Phys. D: Appl. Phys. 39 (2006) 3067-3076.

[6] Z.D. Jia, H.F. Gao, Z.C. Guan, L.M. Wang, J. Yang, Study on hydrophobicity transfer of RTV coatings based on a modification of absorption and cohesion theory, IEEE Trans. Dielectr. Electr. Insul. 13 (2006) 1317-1324.

[7] M. Amin, M. Akbar, S. Amin, Hydrophobicity of silicone rubber used for outdoor insulation (An overview), Rev. Adv. Mater. Sci. 16 (2007) 10-26.

[8] Z.D. Jia, S. Fang, H.F. Gao, Z.C. Guan, L.M. Wang, Z.H. Xu, Development of RTV silicone coatings in China: Overview and bibliography, IEEE Trans. Dielectr. Electr. Insul. 24 (2008) 28-41.

[9] E.A. Cherney, R.S. Gorur, RTV silicone rubber coatings for outdoor insulators, IEEE Trans. Dielectr. Electr. Insul. 6 (1999) 605-611.

[10] R. Hackam, Outdoor HV composite polymeric insulators, IEEE Trans. Dielectr. Electr. Insul. 6 (1999) 557-585.

[11] A.N. Jahromi, E.A. Cherney, S.H. Jayaram, L.C. Simon, Aging characteristics of RTV silicone rubber insulator coatings, IEEE Trans. Dielectr. Electr. Insul. 15 (2008) 444-452.

[12] S. Chandrasekar, R. Sarathi, M.G. Danikas, Analysis of surface degradation of silicone rubber insulation due to tracking under different voltage profiles, Electr. Eng. 89 (2007) 489-501.

[13] M. Fernando, S.M. Gubanski, Ageing of Silicone Rubber Insulators in Coastal and Inland Tropical Environment, IEEE Trans. Dielectr. Electr. Insul. 17 (2010) 326-333.

[14] H. Homma, C.L. Mirley, J. Ronzello, S.A. Boggs, Field and laboratory aging of RTV silicone insulator coatings, Ieee T Power Deliver 15 (2000) 1298-1303.

[15] E.R. Beach, G.W. Tormoen, J. Drelich, R. Han, Pull-off force measurements between rough surfaces by atomic force microscopy, J. Colloid. Interf. Sci. 247 (2002) 84-99.

[16] L. Yan, W. Jing, L. Xidong, L. Yingyan, Adhesion force measurement of electrical insulating materials by atomic force microscopy, IEEE. Power Engineering and Automation Conference (PEAM) (2012) 1-5. 
[17] N. Wang, D.S. Xiong, M.T. Li, Y.L. Deng, Y. Shi, K. Wang, Superhydrophobic surface on steel substrate and its anti-icing property in condensing conditions, Appl. Surf. Sci. 355 (2015) 226-232.

[18] S. Farhadi, M. Farzaneh, S.A. Kulinich, Anti-icing performance of superhydrophobic surfaces, Appl. Surf. Sci. 257 (2011) 6264-6269.

[19] Y. Liu, Y. Bai, J.F. Jin, L.M. Tian, Z.W. Han, L.Q. Ren, Facile fabrication of biomimetic superhydrophobic surface with anti-frosting on stainless steel substrate, Appl. Surf. Sci. 355 (2015) 1238-1244.

[20] T. Jing, Y. Kim, S. Lee, D. Kim, J. Kim, W. Hwang, Frosting and defrosting on rigid superhydrohobic surface, Appl. Surf. Sci. 276 (2013) 37-42.

[21] E. Aljallis, M.A. Sarshar, R. Datla, V. Sikka, A. Jones, C.H. Choi, Experimental study of skin friction drag reduction on superhydrophobic flat plates in high Reynolds number boundary layer flow, Phys. Fluids. 25 (2013) 025103-025104.

[22] S. Lyu, D.C. Nguyen, D. Kim, W. Hwang, B. Yoon, Experimental drag reduction study of super-hydrophobic surface with dual-scale structures, Appl. Surf. Sci. 286 (2013) 206-211

[23] W. Barthlott, C. Neinhuis, Purity of the sacred lotus, or escape from contamination in biological surfaces, Planta 202 (1997) 1-8.

[24] R. Furstner, W. Barthlott, C. Neinhuis, P. Walzel, Wetting and self-cleaning properties of artificial superhydrophobic surfaces, Langmuir 21 (2005) 956-961.

[25] X.Y. Fu, X.H. He, Fabrication of super-hydrophobic surfaces on aluminum alloy substrates, Appl. Surf. Sci. 255 (2008) 1776-1781.

[26] Y.H. Liu, X.K. Wang, J.B. Luo, X.C. Lu, Fabrication and tribological properties of super-hydrophobic surfaces based on porous silicon, Appl. Surf. Sci. 255 (2009) 9430-9438.

[27] L. Zhai, F.C. Cebeci, R.E. Cohen, M.F. Rubner, Stable superhydrophobic coatings from polyelectrolyte multilayers, Nano Lett. 4 (2004) 1349-1353.

[28] N.J. Shirtcliffe, G. McHale, M.I. Newton, G. Chabrol, C.C. Perry, Dual-scale roughness produces unusually water-repellent surfaces, Adv. Mater. 16 (2004) 1929-1932.

[29] M.K. Sarkar, K. Bal, F.E. He, J.T. Fan, Design of an outstanding super-hydrophobic surface by electro-spinning, Appl. Surf. Sci. 257 (2011) 7003-7009.

[30] M.F. Zhu, W.W. Zuo, H. Yu, W. Yang, Y.M. Chen, Superhydrophobic surface directly created by electrospinning based on hydrophilic material, J. Mater. Sci. 41 (2006) 3793-3797.

[31] Q.H. Wang, W.X. Hou, Y.M. Zhang, Superhydrophobic and luminescent methylsilicone resin film, Appl. Surf. Sci. 256 (2009) 664-667.

[32] Z.J. Wei, W.L. Liu, D. Tian, C.L. Xiao, X.Q. Wang, Preparation of lotus-like superhydrophobic fluoropolymer films, Appl. Surf. Sci. 256 (2010) 3972-3976.

[33] I. Woodward, W.C.E. Schofield, V. Roucoules, J.P.S. Badyal, Super-hydrophobic surfaces produced by plasma fluorination of polybutadiene films, Langmuir 19 (2003) 3432-3438.

[34] J. Li, Z.Y. Huang, F.P. Wang, X.Z. Yan, Y. Wei, One-step preparation of transparent superhydrophobic coatings using atmospheric arc discharge, Appl. Phys. Lett. 107 (2015) 093118-093119.

[35] S.A. Mahadik, M.S. Kavale, S.K. Mukherjee, A.V. Rao, Transparent Superhydrophobic silica coatings on glass by sol-gel method, Appl. Surf. Sci. 257 (2010) 333-339.

[36] A.V. Rao, S.S. Latthe, D.Y. Nadargi, H. Hirashima, V. Ganesan, Preparation of MTMS based transparent superhydrophobic silica films by sol-gel method, J. Colloid. Interf. Sci. 332 (2009) 484-490.

[37] Z.G. Guo, W.M. Liu, B.L. Su, Superhydrophobic surfaces: From natural to biomimetic to functional, J. Colloid. Interf. Sci. 353 (2011) 335-355. 
[38] L. Feng, S.H. Li, Y.S. Li, H.J. Li, L.J. Zhang, J. Zhai, Y.L. Song, B.Q. Liu, L. Jiang, D.B. Zhu, Super-hydrophobic surfaces: From natural to artificial, Adv. Mater. 14 (2002) 1857-1860.

[39] M. Miwa, A. Nakajima, A. Fujishima, K. Hashimoto, T. Watanabe, Effects of the surface roughness on sliding angles of water droplets on superhydrophobic surfaces, Langmuir 16 (2000) 5754-5760.

[40] M.A. Samaha, H.V. Tafreshi, M. Gad-el-Hak, Modeling drag reduction and meniscus stability of superhydrophobic surfaces comprised of random roughness, Phys. Fluids. 23 (2011) 012001-012002.

[41] D.K.Owens, R.C.Wendt, Estimation of the surface free energy of polymers, J. Appl. Polym. Sci. 13 (1969) 1741-1747.

[42] M. Stueber, L. Niederberger, F. Danneil, H. Leiste, S. Ulrich, A. Welle, M. Marin, H. Fischer, Surface topography, surface energy and wettability of magnetron-sputtered amorphous carbon (a-c) films and their relevance for platelet adhesion, Adv. Eng. Mater. 9 (2007) 1114-1122.

[43] A.B.D. Cassie, S. Baxter, Wettability of porous surfaces, Transactions of the Faraday Society 40 (1944) 546-551.

[44] H.Y. Dong, M.J. Cheng, Y.J. Zhang, H. Wei, F. Shi, Extraordinary drag-reducing effect of a superhydrophobic coating on a macroscopic model ship at high speed, J. Mater. Chem. A. 1 (2013) 5886-5891.

[45] M.H. Nazemi, V. Hinrichsen, Experimental Investigations on Water Droplet Oscillation and Partial Discharge Inception Voltage on Polymeric Insulating Surfaces under the Influence of AC Electric Field Stress, IEEE Trans. Dielectr. Electr. Insul. 20 (2013) 443-453.

[46] H.F. Gao, Z.D. Jia, Y.K. Mao, Z.C. Guan, L.M. Wang, Effect of hydrophobicity on electric field distribution and discharges along various wetted hydrophobic surfaces, IEEE Trans. Dielectr. Electr. Insul. 15 (2008) $435-443$

[47] K. Takeda, A. Nakajima, Y. Murata, K. Hashimoto, T. Watanabe, Control of water droplets on super-hydrophobic surfaces by static electric field, Jpn J Appl Phys 141 (2002) 287-291.

[48] M. Sakai, H. Kono, A. Nakajima, H. Sakai, M. Abe, A. Fujishima, Water Droplets' Internal Fluidity during Horizontal Motion on a Superhydrophobic Surface with an External Electric Field, Langmuir 26 (2010) 1493-1495. 